\title{
Analysis of a finite matrix with an inhomogeneous circular inclusion subjected to a non-uniform eigenstrain
}

\author{
Biao Wang ${ }^{1}$, Wen Zhao $^{2}$, Lifeng $\mathrm{Ma}^{3, *}$ \\ ${ }^{1}$ Department of Mechanical Engineering, City University of Hong Kong, Kowloon, Hong Kong, China \\ ${ }^{2}$ Physical Science and Engineering Division, King Abdullah University of Science and Technology, Thuwal, Saudi Arabia, 23955-6900 \\ ${ }^{3}$ Department of Engineering Mechanics, Xi'an Jiaotong University, Xi'an, China, 710049 \\ 1. Email: biaowang6-c@my.cityu.edu.hk 2. Email: wen.zhao@kaust.edu.sa 3. Email malf@mail.xjtu.edu.cn
}

\begin{abstract}
The mechanical model of eigenstrains could not be always taken as uniform distributions in engineering applications when performing micromechanics analysis of the inclusion-matrix system. In the framework of plane strain, this paper presents the analytical solution to an inhomogeneous circular inclusion with a non-uniform eigenstrain concentrically embedded in a finite matrix. First, the equivalent eigenstrain equation is extended to satisfy the condition of the finite matrix through the equivalent eigenstrain principle. The modified equation is used to transform the inhomogeneous inclusion in a finite matrix into the corresponding homogeneous inclusion. Then, the model of the inhomogeneous circular inclusion is accordingly formulated and the stress and strain distributions are found. Finally, the stresses for the case of the polynomial series distribution of eigenstrains are obtained. The effects of non-uniformity of eigenstrains, the material mismatch and the inclusion size on stress distributions are shown graphically. The results indicate the stiffer inclusion induce the larger stress under the specific eigenstrain distribution. The analytical solutions obtained here also help to predict failure and optimize designs of composite structure.
\end{abstract}

Keywords: Inhomogeneous inclusion, Non-uniform eigenstrain, Finite matrix, Circular inclusion, Polynomial series distribution of eigenstrains.

\section{Introduction}

Inclusion problems have been widely studied due to their fundamental role and practical applications in micromechanics and advanced materials. Along with the intrinsic physical and engineering significance of understating material properties and designing new materials, numerous efforts have been dedicated to investigating inclusions in an infinite matrix. These studies, which can be mainly categorized into homogenous and inhomogeneous inclusion problems, have largely released the assumptions existed in the pioneering works of Eshelby [1-2]. Besides elliptical and ellipsoid inclusions and uniform eigenstrains distributed in inclusions, the studies have considered line [3-5], polygonal (or polyhedral) [6-11] and even arbitrary-shaped [12-18] inclusions and also extended the eigenstrains to non-uniformity [19-23]. A comprehensive review work can be found in literature [24].

In many situations encountered in practical engineering, assuming that the size of matrix is infinite cannot be always sufficiently accurate since the size of inclusions is not always relatively small. For instance, the volume fraction of carbon nanotubes in composites has been raised up to above $20 \%$ in order to improve their relatively low stiffness and strength [25-27]. In the particle-reinforced composite cases, $\mathrm{Al}_{2} \mathrm{O}_{3}$ took up a volume fraction of $50 \%$ in metal matrix composite [28]. In such cases, the boundary effect of matrix has to be considered. Thus, inclusions in a finite matrix draw much interests. For example, Li et.al. and Wang et.al. [29-32] investigated a homogenous circular concentrically surrounded by a circular domain and a spherical inclusion concentrically embedded in a spherical 
matrix. The considered inclusion is prescribed with a uniform eigenstrain and the exterior boundary of the matrix is subjected to the displacement and traction boundary conditions. They obtained the closed-form expressions of Dirichlet-Eshelby and Neumann-Eshelby tensors related to different boundary conditions. Subsequently, based on the elasticity theory of a simplified strain gradient, Gao et.al [33] and Ma et.al [34] tested the similar problems and extended the solutions in the framework of high-order elasticity theory. Mejak [35] studied axisymmetrically placed inclusion in a sphere and found the Dirichlet-Eshelby tensor of an eccentric spherical inclusion through the power series expansion. Research on arbitrarily-shaped inclusion problems studied by Zou [36] is quite significant. In his paper, the authors proposed a general procedure based on the superposition principle, which overcame the unavailability of the Green's function. He also derived the explicit results for a concentric square inclusion and an eccentric circular inclusion. After that, Zou and He [37] used the superposition principle [36] to further analyze an eccentric spherical inclusion with a uniform eigenstrain located in a finite sphere. Chen [38] conducted a numerical investigation of an elliptical inclusion in a finite elliptic plate, considering a uniform eigenstrain in the inclusion and static loading on the outer boundary of matrix. Pan and Yu [39] considered the shape effect of martrix for circular inclusion problem using complex potential method along with conformal mapping. Subsequently, they [40] further extended their work to an arbitrarily-shaped inclusion by assuming that the shape of an inclusion can be replaced with an arbitrarily-shaped polygon. Both works were also verified by simulations.

However, all the researches that we investigated only considered the homogeneous inclusions with the uniform eigenstrain. In this paper, we aim to derive an analytical solution for the inhomogeneous circular inclusion problem with a non-uniform symmetrical eigenstrain concentrically embedded in a circular matrix. Symmetrical eigenstrains exist in problems of point heat source, diffusion of materials and compositional changes [41]. It is an extension to our previous work [42-43] in which the authors dealt with an inhomogeneous inclusion with non-uniform eigenstrains in an infinite matrix. Toward this, a procedure of solving an inhomogeneous arbitrarily-shaped inclusion with non-uniform eigenstrains in a finite matrix is presented. We enrich the equivalent eigenstrain principle and the modified equivalent eigenstrain equation is also derived.

This paper is organized as follows. Section 2 presents an modified equation of equivalent eigenstrain for solving inhomogeneous inclusion problems in a finite matrix. And in Section 3, analytical solutions to the circular inhomogeneous inclusion problem with a non-uniform eigenstrain is provided. We illustrate a typical example in Section 4 to study the inhomogeneous inclusion problem with a polynomial-distributed eigenstrain and its effect of eigenstrain distributions, the material mismatch and the inclusion size on elastic field is also analyzed. Finally, the main conclusions are summarized in Section 5 .

\section{An extended equivalent eigenstrain equation for a finite matrix}

Consider an inhomogeneous arbitrarily-shaped inclusion $\Omega$ prescribed with a non-uniform eigenstrain $\varepsilon_{i j}^{*}$ is embedded into a finite matrix, as shown in Fig. 1(a). The elastic moduli of the matrix and the inhomogeneous inclusion are $C_{i j k l}$ and $C_{i j k l}^{*}$, respectively. The boundary $\partial D$ is subjected to boundary loads $\mathbf{T}$. 


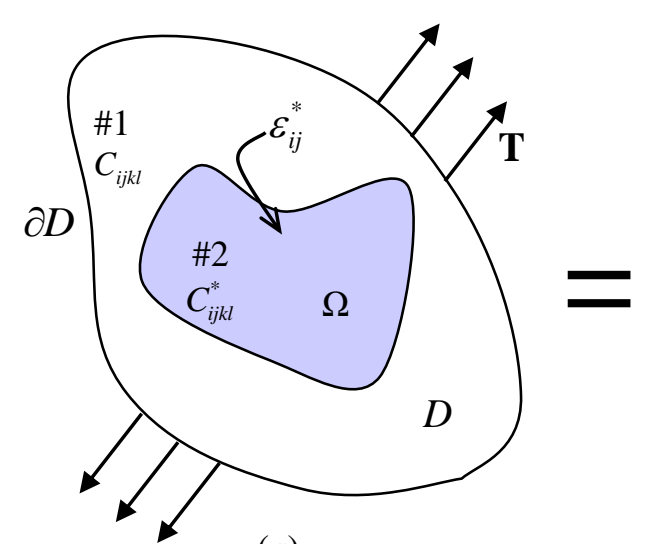

(a)

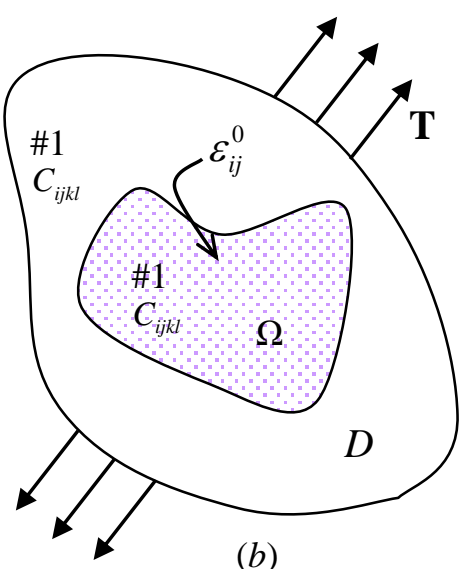

(b)

Fig. 1. (a) The inhomogeneous arbitrarily-shaped inclusion in a finite elastic body subjected to a non-uniform eigenstrain and boundary loads, $(b)$ The corresponding homogeneous inclusion problem. The inhomogeneous inclusion problem in Fig. 1(a) is equivalent to the corresponding homogeneous inclusion problem in Fig. 1(b) via the extended equivalent eigenstrain equation.

Based on the equivalent eigenstrain principle [29], the inhomogeneous inclusion problem in Fig. 1(a) can be transformed into the corresponding homogeneous inclusion problem (Fig. 1 $(b)$ ). But the equivalent eigenstrain $\varepsilon_{i j}^{0}$ equation has to be extended as following,

$$
\varepsilon_{i j}^{0}=S_{i j k l}\left(C_{k l p q}-C_{k l p q}^{*}\right) \varepsilon_{p q}+S_{i j k l} C_{k l p q}^{*} \varepsilon_{p q}^{*}
$$

Where $S_{i j k l}=C_{i j k l}^{-1}$ is the elastic compliance of the matrix, the correction term $\varepsilon_{i j}$ represents the total strain caused by both $\varepsilon_{i j}^{0}$ and $\mathbf{T}$ in the homogenous inclusion $\Omega$. In the following step, we will introduce how to find the relation between $\varepsilon_{i j}, \varepsilon_{i j}^{0}$ and $\mathbf{T}$. The actual stress $\sigma_{i j}$ in Fig. 1(b) can be evaluated by the sum of $\sigma_{i j}^{0}$ and $\sigma_{i j}^{1}$, where $\sigma_{i j}^{0}$ is caused by $\varepsilon_{i j}^{0}$ in an infinite matrix and $\sigma_{i j}^{1}$ satisfies the boundary conditions on $\partial D$ :

$$
\left.\sigma_{i j}^{1}\right|_{\partial D}=-\left.\sigma_{i j}^{0}\right|_{\partial D}+\mathbf{T}
$$

Once the stress $\sigma_{i j}$ is obtained, then, Hooke's law yeilds

$$
\begin{gathered}
\varepsilon_{i j}=\varepsilon_{i j}^{0}+S_{i j k l} \sigma_{k l}, \text { in } \Omega \\
\varepsilon_{i j}=S_{i j k l} \sigma_{k l}, \quad \text { in } D
\end{gathered}
$$

By combining Eqs. (1-4), the equivalent eigenstrain $\varepsilon_{i j}^{0}$, the total stress $\sigma_{i j}$ and the total strain $\varepsilon_{i j}$ can be calculated. Since the original inhomogeneous problem is completely equivalent to the corresponding homogeneous inclusion problem, the elastic field in the origin problem is found. 
On should note that the boundary loads $\mathbf{T}$ are remote loads and $\left.\sigma_{i j}^{1}\right|_{\partial D}=\mathbf{T}$ when the domain of the matrix becomes infinite, which indicates that the problem in a finite domain is directly degenerated into the corresponding problem in an infinite domain. In such case, Eqs. (1) to (4) are consistent with the solutions in the previous work [42].

We here in theory solve the inhomogeneous inclusion with a non-uniform eigenstrain in a finite matrix. It provides a framework to obtain analytical as well as numerical solutions for specific inhomogeneous inclusion problems. In the following, we will use the procedure to derive the analytical solution for an inhomogeneous circular inclusion in a finite matrix.

\section{Analytical solution for an inhomogeneous circular inclusion with a non-uniform symmetrical eigenstrain}

In the framework of plane strain, the inhomogeneous circular inclusion subjected to a non-uniform symmetrical eigenstrain will be analyzed. As shown in Fig. 2(a), a finite circular domain $\left(\rho_{1}<\rho<\rho_{2}\right)$ with the elastic moduli $C_{i j k l}$ concentrically contains an inhomogeneous inclusion $\left(\rho<\rho_{1}\right)$ whose the elastic moduli are $C_{i j k l}^{*}$. Eigenstrain given in the inhomogeneous circular inclusion is assumed to be in the form of $\varepsilon_{i j}^{*}(\rho)=\delta_{i j} \varepsilon^{*}(\rho)$ and zero in the matrix. Fig. 2(b) shows the corresponding homogenous inclusion with the equivalent eigenstrain $\varepsilon_{i j}^{0}$. For plain strain condition and isotropic materials, the components of $C_{i j k l}, C_{i j k l}^{*}, \varepsilon_{i j}^{*}(\rho) \varepsilon_{i j}^{0}$ and the total strain $\varepsilon_{i j}$ in question are expressed as follows,

$$
\begin{aligned}
& {\left[C_{i j k l}\right]=\frac{2 \mu}{1-2 v}\left[\begin{array}{ccc}
1-v & v & 0 \\
v & 1-v & 0 \\
0 & 0 & 1-2 v
\end{array}\right], \quad\left[C_{i j k l}^{*}\right]=\frac{2 \mu^{*}}{1-2 v^{*}}\left[\begin{array}{ccc}
1-v^{*} & v^{*} & 0 \\
v^{*} & 1-v^{*} & 0 \\
0 & 0 & 1-2 v^{*}
\end{array}\right]} \\
& {\left[\begin{array}{c}
\varepsilon_{i j}^{*}
\end{array}\right]=\left[\begin{array}{c}
\varepsilon_{\theta \theta}^{*} \\
\varepsilon_{r r}^{*} \\
\varepsilon_{r \theta}^{*}
\end{array}\right],\left[\varepsilon_{i j}^{0}\right]=\left[\begin{array}{c}
\varepsilon_{\theta \theta}^{0} \\
\varepsilon_{r r}^{0} \\
\varepsilon_{r \theta}^{0}
\end{array}\right],\left[\varepsilon_{i j}\right]=\left[\begin{array}{c}
\varepsilon_{\theta \theta} \\
\varepsilon_{r r} \\
\varepsilon_{r \theta}
\end{array}\right]}
\end{aligned}
$$




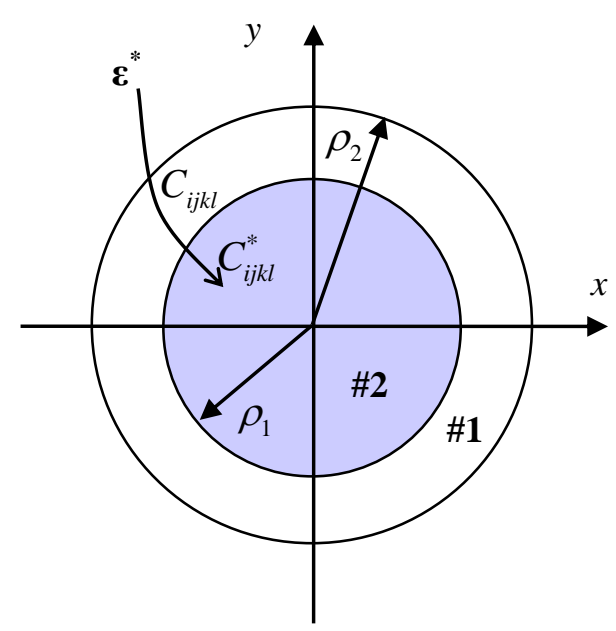

(a)

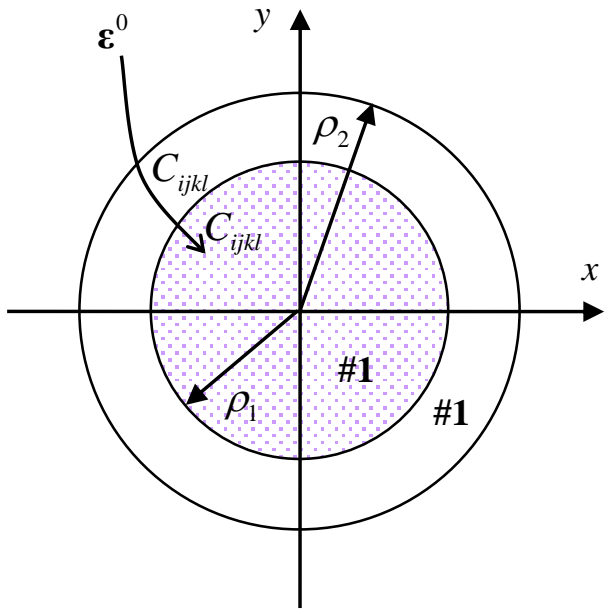

(b)

Fig. 2. (a) The inhomogeneous circular inclusion problem in a finite circular domain, (b) The corresponding homogeneous circular inclusion problem. The original problem in Fig. 2(a) is transformed into the homogeneous inclusion problem in Fig. 2(b).

\subsection{Homogeneous circular inclusion}

Here, we first present the relation between the total strain $\varepsilon_{i j}$ and the equivalent eigenstrain $\varepsilon_{i j}^{0}$ before solving the extended equivalent equation by studing the homogeneous inclusion problem in Fig. $2(b)$. Assume the equivalent eigenstrain $\varepsilon_{i j}^{0}(\rho)$ is distributed in the form of $\varepsilon_{i j}^{0}(\rho)=\delta_{i j} \varepsilon^{0}(\rho)$.

As discussed in Section 2, the problem can be decomposed into two sub-problems. For the homogeneous circular inclusion with the same eigenstrain in an infinite matrix, the stress and strain components at any point $(x, y)$ outside the circular inclusion, i.e., $\rho=\sqrt{x^{2}+y^{2}}>\rho_{1}$ are [43],

$$
\begin{aligned}
& {\left[\begin{array}{c}
\sigma_{\theta \theta}^{0}(\rho) \\
\sigma_{r r}^{0}(\rho) \\
\sigma_{r \theta}^{0}(\rho)
\end{array}\right]=\frac{2 \mu}{(1-v) \rho^{2}} \int_{0}^{\rho_{1}} \varepsilon_{0}\left(\rho_{0}\right) \rho_{0} d \rho_{0}\left[\begin{array}{c}
1 \\
-1 \\
0
\end{array}\right],\left(\rho=\sqrt{x^{2}+y^{2}}>\rho_{1}\right)} \\
& {\left[\begin{array}{c}
\varepsilon_{\theta \theta}^{0}(\rho) \\
\varepsilon_{r r}^{0}(\rho) \\
\varepsilon_{r \theta}^{0}(\rho)
\end{array}\right]=\frac{1}{(1-v) \rho^{2}} \int_{0}^{\rho_{1}} \varepsilon_{0}\left(\rho_{0}\right) \rho_{0} d \rho_{0}\left[\begin{array}{c}
1 \\
-1 \\
0
\end{array}\right],\left(\rho=\sqrt{x^{2}+y^{2}}>\rho_{1}\right)}
\end{aligned}
$$

And at those points inside the inclusion, i.e., $\rho<\rho_{1}$, they are expressed as,

$$
\left[\begin{array}{c}
\sigma_{\theta \theta}^{0}(\rho) \\
\sigma_{r r}^{0}(\rho) \\
\sigma_{r \theta}^{0}(\rho)
\end{array}\right]=-\frac{2 \mu}{(1-v) \rho^{2}} \int_{0}^{\rho} \varepsilon_{0}\left(\rho_{0}\right) \rho_{0} d \rho_{0}\left[\begin{array}{l}
1 \\
1 \\
0
\end{array}\right],\left(\rho<\rho_{1}\right)
$$




$$
\left[\begin{array}{c}
\varepsilon_{\theta \theta}^{0}(\rho) \\
\varepsilon_{r r}^{0}(\rho) \\
\varepsilon_{r \theta}^{0}(\rho)
\end{array}\right]=-\frac{(1-2 v)}{(1-v) \rho^{2}} \int_{0}^{\rho} \varepsilon_{0}\left(\rho_{0}\right) \rho_{0} d \rho_{0}\left[\begin{array}{l}
1 \\
1 \\
0
\end{array}\right]+\left[\begin{array}{c}
\varepsilon_{0}(\rho) \\
\varepsilon_{0}(\rho) \\
0
\end{array}\right],\left(\rho<\rho_{1}\right)
$$

For the specific boundary value problem, the boundary loads are given by,

$$
\left.\sigma_{r r}^{1}\right|_{\rho=\rho_{2}}=\left.\sigma_{r r}^{0}\right|_{\rho=\rho_{2}}=-\frac{2 \mu}{(1-v) \rho_{2}^{2}} \int_{0}^{\rho_{1}} \varepsilon_{0}\left(\rho_{0}\right) \rho_{0} d \rho_{0},\left.\quad \sigma_{r \theta}^{1}\right|_{\rho=\rho_{2}}=0
$$

Then, its stress and strain components in Fig. 2(b) are obtained:

$$
\begin{aligned}
& {\left[\begin{array}{c}
\sigma_{\theta \theta}^{1}(\rho) \\
\sigma_{r r}^{1}(\rho) \\
\sigma_{r \theta}^{1}(\rho)
\end{array}\right]=\frac{2 \mu}{(1-v) \rho_{2}^{2}} \int_{0}^{\rho_{1}} \varepsilon_{0}\left(\rho_{0}\right) \rho_{0} d \rho_{0}\left[\begin{array}{l}
1 \\
1 \\
0
\end{array}\right]} \\
& {\left[\begin{array}{l}
\varepsilon_{\theta \theta}^{1}(\rho) \\
\varepsilon_{r r}^{1}(\rho) \\
\varepsilon_{r \theta}^{1}(\rho)
\end{array}\right]=\frac{(1-2 v)}{(1-v) \rho_{2}^{2}} \int_{0}^{\rho_{1}} \varepsilon_{0}\left(\rho_{0}\right) \rho_{0} d \rho_{0}\left[\begin{array}{l}
1 \\
1 \\
0
\end{array}\right]}
\end{aligned}
$$

By superposing the solutions above, the stress and strain distribution in Fig. 2(a) is given by as follows. For $\rho_{1}<\rho<\rho_{2}$,

$$
\begin{aligned}
& {\left[\begin{array}{l}
\sigma_{\theta \theta}(\rho) \\
\sigma_{r r}(\rho) \\
\sigma_{r \theta}(\rho)
\end{array}\right]=\frac{2 \mu}{(1-v) \rho^{2}} \int_{0}^{\rho_{1}} \varepsilon_{0}\left(\rho_{0}\right) \rho_{0} d \rho_{0}\left[\begin{array}{c}
1 \\
-1 \\
0
\end{array}\right]+\frac{2 \mu}{(1-v) \rho_{2}^{2}} \int_{0}^{\rho_{1}} \varepsilon_{0}\left(\rho_{0}\right) \rho_{0} d \rho_{0}\left[\begin{array}{l}
1 \\
1 \\
0
\end{array}\right],\left(\rho_{1}<\rho<\rho_{2}\right)} \\
& {\left[\begin{array}{l}
\varepsilon_{\theta \theta}(\rho) \\
\varepsilon_{r r}(\rho) \\
\varepsilon_{r \theta}(\rho)
\end{array}\right]=\frac{1}{(1-v) \rho^{2}} \int_{0}^{\rho_{1}} \varepsilon_{0}\left(\rho_{0}\right) \rho_{0} d \rho_{0}\left[\begin{array}{c}
1 \\
-1 \\
0
\end{array}\right]+\frac{(1-2 v)}{(1-v) \rho_{2}^{2}} \int_{0}^{\rho_{1}} \varepsilon_{0}\left(\rho_{0}\right) \rho_{0} d \rho_{0}\left[\begin{array}{l}
1 \\
1 \\
0
\end{array}\right],\left(\rho_{1}<\rho<\rho_{2}\right)}
\end{aligned}
$$

For $\rho<\rho_{1}$,

$$
\begin{gathered}
{\left[\begin{array}{c}
\sigma_{\theta \theta}(\rho) \\
\sigma_{r r}(\rho) \\
\sigma_{r \theta}(\rho)
\end{array}\right]=-\frac{2 \mu}{(1-v) \rho^{2}} \int_{0}^{\rho} \varepsilon_{0}\left(\rho_{0}\right) \rho_{0} d \rho_{0}\left[\begin{array}{l}
1 \\
1 \\
0
\end{array}\right]+\frac{2 \mu}{(1-v) \rho_{2}^{2}} \int_{0}^{\rho_{1}} \varepsilon_{0}\left(\rho_{0}\right) \rho_{0} d \rho_{0}\left[\begin{array}{l}
1 \\
1 \\
0
\end{array}\right],\left(\rho<\rho_{1}\right)} \\
{\left[\begin{array}{l}
\varepsilon_{\theta \theta}(\rho) \\
\varepsilon_{r r}(\rho) \\
\varepsilon_{r \theta}(\rho)
\end{array}\right]=-\frac{(1-2 v)}{(1-v) \rho^{2}} \int_{0}^{\rho} \varepsilon_{0}\left(\rho_{0}\right) \rho_{0} d \rho_{0}\left[\begin{array}{l}
1 \\
1 \\
0
\end{array}\right]} \\
+\frac{(1-2 v)}{(1-v) \rho_{2}^{2}} \int_{0}^{\rho_{1}} \varepsilon_{0}\left(\rho_{0}\right) \rho_{0} d \rho_{0}\left[\begin{array}{l}
1 \\
1 \\
0
\end{array}\right]+\left[\begin{array}{c}
\varepsilon_{0}(\rho) \\
\varepsilon_{0}(\rho) \\
0
\end{array}\right],\left(\rho<\rho_{1}\right)
\end{gathered}
$$

The stress and strain fields of homogeneous inclusion in a finite domain are now derived. It is clearly shown that the solution is a further extended and also can be degenerated into the case in our previous researches [43] simply by letting $\rho_{2} \rightarrow+\infty$.

\subsection{Inhomogeneous circular inclusion}

Via the modified equivalent eigenstrain equation (see Eq. (1)), $\varepsilon_{i j}^{0}$ in Fig. 2(b) satisfies, 


$$
C_{i j k l} \varepsilon_{k l}^{0}=\left(C_{i j k l}-C_{i j k l}^{*}\right) \varepsilon_{k l}+C_{i j k l}^{*} \varepsilon_{k l}^{*}, \quad\left(\rho<\rho_{1}\right)
$$

Due to the symmetry of both geometry and eigenstrain distribution, the total strain $\varepsilon_{i j}(\rho)$ and the equivalent strain $\varepsilon_{i j}^{0}(\rho)$ must have the form of $\varepsilon_{i j}(\rho)=\delta_{i j} \varepsilon(\rho), \varepsilon_{i j}^{0}(\rho)=\delta_{i j} \varepsilon_{0}(\rho)$.

Substituting Eqs. (5), (16) into Eq. (17), we find

$$
\varepsilon_{0}(\rho)+\chi \frac{1}{\rho^{2}} \int_{0}^{\rho} \varepsilon_{0}\left(\rho_{0}\right) \rho_{0} d \rho_{0}+c_{1}=\varepsilon^{*}(\rho), \quad\left(\rho<\rho_{1}\right)
$$

Where

$$
\begin{aligned}
& \chi=\frac{\mu\left(1-2 v^{*}\right)-\mu^{*}(1-2 v)}{\mu^{*}(1-v)} \\
& c_{1}=-\frac{\chi}{\rho_{2}^{2}} \int_{0}^{\rho_{1}} \varepsilon_{0}\left(\rho_{0}\right) \rho_{0} d \rho_{0}
\end{aligned}
$$

Its solution is

$$
\varepsilon_{0}(\rho)=\frac{1}{\rho^{\chi+2}}\left\{\int\left[\rho^{\chi} \frac{d}{d \rho}\left(\rho^{2} \varepsilon^{*}(\rho)\right)-2 c_{1} \rho^{\chi+1}\right] d \rho+c_{2}\right\}, \quad\left(\rho<\rho_{1}\right)
$$

The constant $c_{2}$ can be determined by inserting Eq. (20) into Eq. (18). Now that the equivalent strain $\varepsilon_{i j}^{0}(\rho)$ is derived, the stress and the strain distributions in both inhomogeneous inclusion and matrix zone can be calculated by Eqs. (13) to (16).

It is worthy to note that the constant $c_{1}$ indicates the boundary effect of the matrix and $\chi$ represents the disparity of material properties between the matrix and the inclusion. As seen in Eq. (19), $c_{1}$ tends to zero when the radius of matrix $\rho_{2}$ extends to infinity. In such case, the solutions obtained above are degenerated to the solution for the inhomogeneous inclusion problem in an infinite elastic plane [43]. Moreover, when the inclusion and the matrix share the same material, thus, $\chi$ becomes zero, the solutions are directly degenerated into those for homogeneous inclusion problems, which prove our conclusions in Section 2.

\section{A representative example: Polynomial series distributions of eigenstrains}

In the previous two sections, the elastic field to both homogeneous and inhomogeneous circular inclusion with a non-uniform eigenstrain were obtained. And, from the perspective of understanding material properties and designing new material structures, the elastic interactions between inclusions and matrix has to be fully understand. Thus, in this section, we will apply the solutions to consider typical polynomial series distributions of eigenstrains to analyze the influence of eigenstrain distributions, the material mismatch and the size of the inclusion on stress distribution.

Assuming the eigenstrains are distributed in terms of polynomial series as,

$$
\varepsilon^{*}(\rho)=\sum_{n=0}^{N} A_{n}\left(\rho / \rho_{1}\right)^{n}, \quad\left(\rho<\rho_{1}\right)
$$

The equivalent eigenstrain $\varepsilon_{i j}^{0}$ is solved by Eq. (20), 


$$
\varepsilon_{0}(\rho)=\sum_{n=0}^{N} A_{n}\left(\frac{n+2}{n+2+\chi} \frac{\rho^{n}}{\rho_{1}^{n}}-\frac{2}{n+2+\chi} \frac{H}{H+1}\right), \quad\left(\rho<\rho_{1}\right)
$$

Where $H=-\frac{\chi}{\chi+2} \frac{\rho_{1}^{2}}{\rho_{2}^{2}}$.

Inserting Eq. (22) into Eq. (13), we obtain the stress distribution in the zone $\rho_{1}<\rho<\rho_{2}$,

$$
\left\{\begin{array}{l}
\sigma_{r r}(\rho)=\sum_{n=0}^{N} \frac{2 A_{n} \mu \rho_{1}^{2}}{(1-v)(n+2+\chi)(H+1)}\left(-\frac{1}{\rho^{2}}+\frac{1}{\rho_{2}^{2}}\right) \\
\sigma_{\theta \theta}(\rho)=\sum_{n=0}^{N} \frac{2 A_{n} \mu \rho_{1}^{2}}{(1-v)(n+2+\chi)(H+1)}\left(\frac{1}{\rho^{2}}+\frac{1}{\rho_{2}^{2}}\right) \\
\sigma_{r \theta}(\rho)=0
\end{array}\right.
$$

And the stress components in the zone $\rho<\rho_{1}$ by Eq. (15) are

$$
\left\{\begin{array}{l}
\sigma_{r r}(\rho)=\sigma_{\theta \theta}(\rho)=\sum_{n=0}^{N} \frac{2 A_{n} \mu}{(1-v)(n+2+\chi)}\left(\frac{1}{H+1} \frac{\rho_{1}^{2}}{\rho_{2}^{2}}-\frac{\rho^{n}}{\rho_{1}^{n}}+\frac{H}{H+1}\right) \\
\sigma_{r \theta}(\rho)=0
\end{array}\right.
$$

It can be found from Eqs. (23) and (24) that the value of radial stress inside the inclusion is equal to the hoop stress at the same position, while they are not equal outside.

For the sake of simplification and clarity, in all cases below, we choose the material such that $v=v^{*}=0.3$. Moreover, soft inclusion $\mu / \mu^{*}=10$ is set in the first case, and the others include homogeneous inclusion $\mu / \mu^{*}=1$ and stiff inclusion $\mu / \mu^{*}=1 / 10$. Corresponding to each material parameter, $\varepsilon^{*}(\rho)=1, \varepsilon^{*}(\rho)=\rho / \rho_{1}, \varepsilon^{*}(\rho)=\left(\rho / \rho_{1}\right)^{2}$ represent the uniform, linear and nonlinear eigenstrain distribution, respectively.

Fig. 3 shows the dimensionless radial stress $\bar{\sigma}_{r r}=\sigma_{r r} / \mu$ and hoop stress $\bar{\sigma}_{\theta \theta}=\sigma_{\theta \theta} / \mu$ against dimensionless radius $\rho / \rho_{2}$ for these three cases when $\rho_{1} / \rho_{2}=0.2$. It can be observed that:

(i) The radial stress $\bar{\sigma}_{r r}$ is continuous at the interface of the inclusion and the matrix, and $\bar{\sigma}_{r r}(1)=0$, while the hoop stress $\bar{\sigma}_{\theta \theta}$ is discontinuous at the position and $\bar{\sigma}_{\theta \theta}>0$ in the matrix;

(ii) Under given eigenstrain distribution, the stiffer inclusion it is, the bigger amplitude stress distribution occurs;

(iii) The curves of the stress distribution are polynomials of degree $n$ inside the inclusion and parabolas outside the inclusion.

(iv) The radial and hoop stress keeps constant inside the inclusion and variable outside the inclusion under the case of $n=0$, which shows the boundary effect of matrix. This result is consistent with the result [44]. 

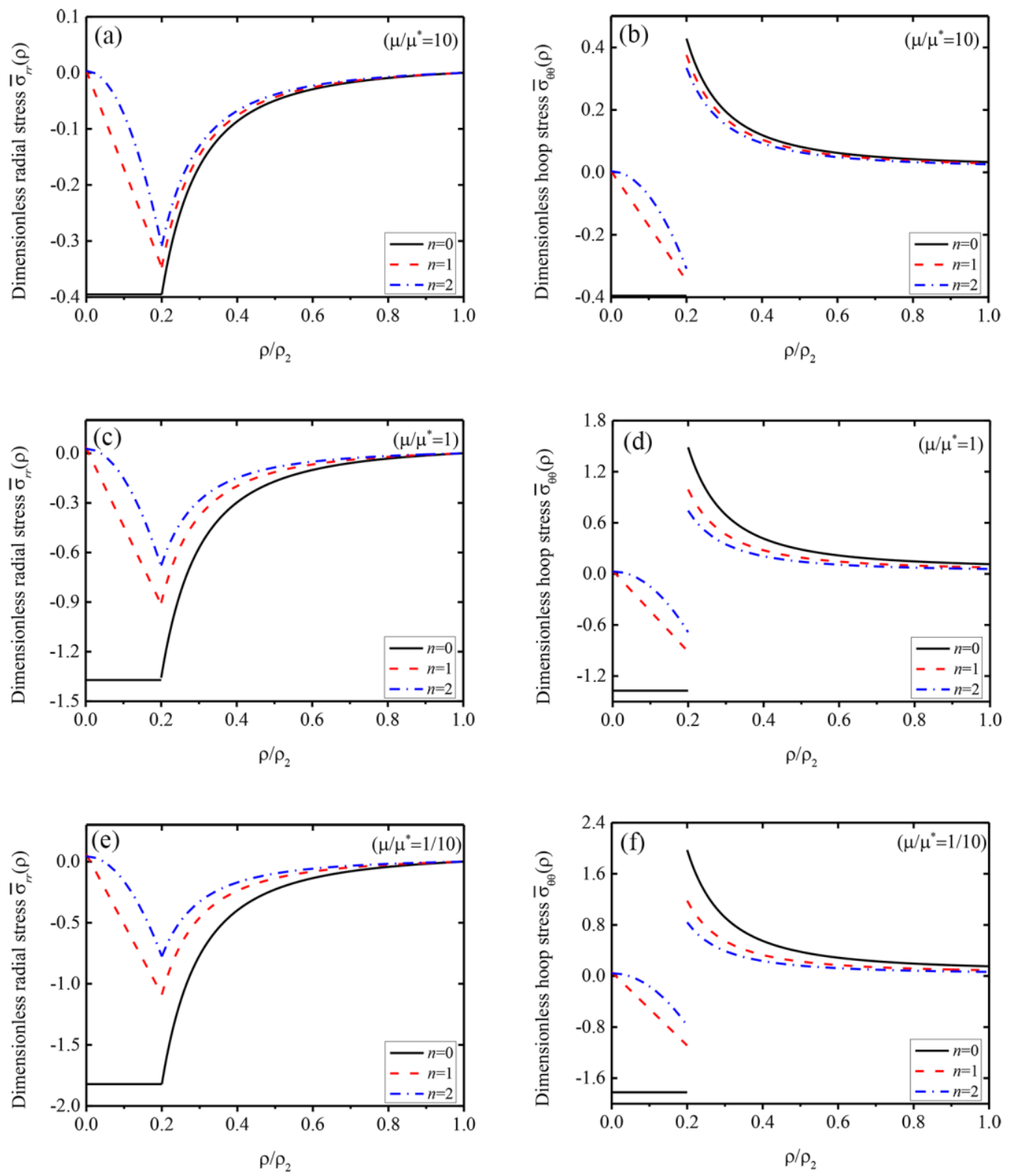

Fig. 3. The dimensionless radial and hoop stress distribution along radius corresponding to soft inclusion $\mu / \mu^{*}=10$, homogeneous inclusion $\mu / \mu^{*}=1$ and stiff inclusion $\mu / \mu^{*}=1 / 10$ when $\rho_{1} / \rho_{2}=0.2$.

When the inclusion covers a volume fraction of 50\%, i.e., $\rho_{1} / \rho_{2}=0.5$, the dimensionless radial stresses $\bar{\sigma}_{r r}=\sigma_{r r} / \mu$ and hoop stress $\bar{\sigma}_{\theta \theta}=\sigma_{\theta \theta} / \mu$ are shown in Fig. 4 . Similar trends can be seen but the discontinuity of the hoop stress at the interface of the inclusion and the matrix increases, thus enhancing the boundary effect as the fraction of inclusion becomes larger. 

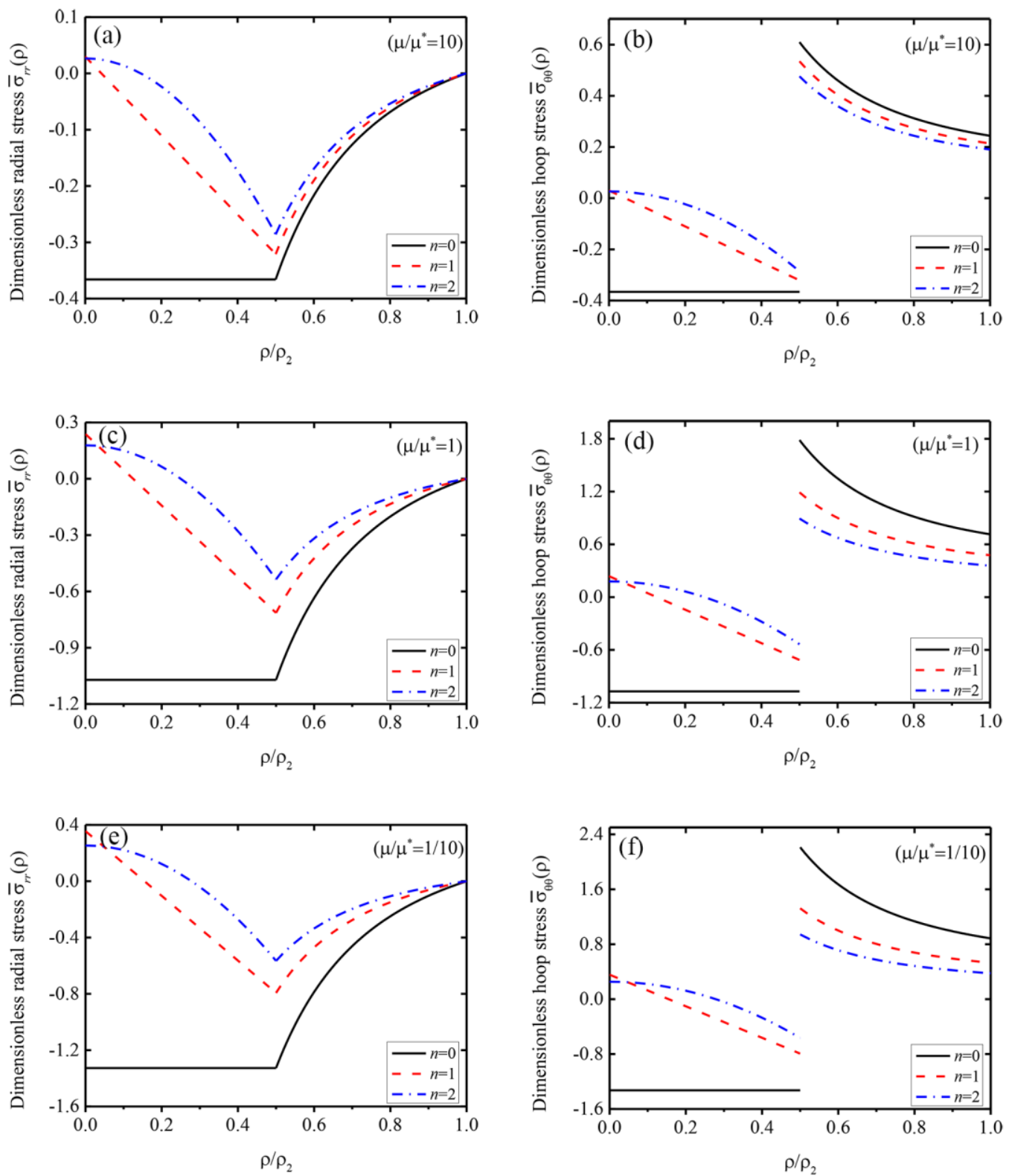

Fig. 4. The dimensionless radial and hoop stress distribution along radius corresponding to soft inclusion $\mu / \mu^{*}=10$, homogeneous inclusion $\mu / \mu^{*}=1$ and stiff inclusion when $\rho_{1} / \rho_{2}=0.5$.

\section{Conclusion}

In this paper, we mainly study the inhomogeneous circular inclusion problem with a non-uniform eigenstrain in a finite matrix, which is motivated by the researches in studying the finite matrix containing the homogeneous inclusion with uniform eigenstrains. First, we develop the procedure to deal with the general cases of the inhomogeneous inclusion problems based on the equivalent eigenstrain principle. Its equation is then extended to unify both cases of the finite and infinite matrix. Then, the elastic fields of both homogeneous and inhomogeneous circular inclusion problem are directly obtained. The two key parameters, affected by the finite boundary of the matrix and the material difference, respectively, have been found.

Besides, we use a typical example to illustrate the effects of eigenstrain distributions, material 
mismatch and the inclusion ratio on stress fields. We find that the hoop stress at the interface is discontinuous, and the discontinuity is enhanced because of increasement on the stiffness and inclusion domain.

This paper only considers the two-dimensional inclusion problem in the framework of plane strain, but three-dimensional cases with non-uniform eigenstrains also surely can be analyzed through the same way. All the results derived in this paper can help engineers to design and evaluate materials and predict their failures.

\section{References}

[1] Eshelby J D (1957) The determination of the elastic field of an ellipsoidal inclusion, and related problems. Proceedings of the Royal Society of London. Series A. Mathematical and Physical Sciences 241(1226): 376-396.

[2] Eshelby J D (1959) The elastic field outside an ellipsoidal inclusion. Proceedings of the Royal Society of London. Series A. Mathematical and Physical Sciences 252(1271): 561-569.

[3] Ma L, Wang B and Korsunsky A M (2018) Complex variable formulation for a rigid line inclusion interacting with a generalized singularity. Archive of Applied Mechanics 88(4): 613-627.

[4] Ma L and Qiu Y and Zhang Y, et al. (2019) General solution for inhomogeneous line inclusion with non-uniform eigenstrain. Archive of Applied Mechanics: 1-19.

[5] Jobin T, Ramji M and Khaderi S (2019) Numerical evaluation of the interaction of rigid line inclusions using strain intensity factors. International Journal of Mechanical Sciences 153: $10-20$.

[6] Jin X, Keer L M and Wang Q. (2009). Analytical solution for the stress field of Eshelby's inclusion of polygonal shape. In: ASME/STLE 2009 International Joint Tribology Conference, pp. 487-489.

[7] Xu B and Wang M (2005) Special properties of Eshelby tensor for a regular polygonal inclusion. Acta Mechanica Sinica 21(3): 267-271.

[8] Sun L, Xu K and Pan E (2012) Inclusion of arbitrary polygon with graded eigenstrain in an anisotropic piezoelectric full plane. International Journal of Solids and Structures 49(13): 1773-1785.

[9] Yue Y and Xu K and Chen Q, et al. (2015) Eshelby problem of an arbitrary polygonal inclusion in anisotropic piezoelectric media with quadratic eigenstrains. Acta Mechanica 226(7): 2365-2378.

[10] Chiang C-R (2016) Problems of polygonal inclusions in orthotropic materials with due consideration on the stresses at corners. Archive of Applied Mechanics 86(5): 769-785.

[11] Nozaki H and Taya M (2001) Elastic fields in a polyhedral inclusion with uniform eigenstrains and related problems. Journal of Applied mechanics 68(3): 441-452.

[12] Ru C (2003) Eshelby inclusion of arbitrary shape in an anisotropic plane or half-plane. Acta mechanica 160(3-4): 219-234.

[13] Nakasone Y, Nishiyama H and Nojiri T (2000) Numerical equivalent inclusion method: a new computational method for analyzing stress fields in and around inclusions of various shapes. Materials Science and Engineering: A 285(1-2): 229-238.

[14] Dong C, Lo S and Cheung Y (2003) Stress analysis of inclusion problems of various shapes in an infinite anisotropic elastic medium. Computer methods in applied mechanics and engineering 192(5-6): 683-696.

[15] Wang J and Michelitsch T M and Gao H, et al. (2005) On the solution of the dynamic Eshelby 
problem for inclusions of various shapes. International journal of solids and structures $42(2)$ : 353-363.

[16] Wang X and Schiavone P (2015) Eshelby's problem for infinite, semi-infinite and two bonded semi-infinite laminated anisotropic thin plates. Archive of Applied Mechanics 85(5): 573-585.

[17] Zhou Q and Jin X and Wang Z, et al. (2015) Numerical implementation of the equivalent inclusion method for 2D arbitrarily shaped inhomogeneities. Journal of Elasticity 118(1): 39-61.

[18] Wang X and Gao X-L (2011) On the uniform stress state inside an inclusion of arbitrary shape in a three-phase composite. Zeitschrift für angewandte Mathematik und Physik 62(6): 1101-1116.

[19] Nie G and Chan C and Luo L, et al. (2009) Non-uniform eigenstrain induced anti-plane stress field in an elliptic inhomogeneity embedded in anisotropic media with a single plane of symmetry. Acta mechanica 206(1-2): 23-37.

[20] Nie G and Guo L and Chan C, et al. (2009) Non-uniform eigenstrain induced stress field in an elliptic inhomogeneity embedded in orthotropic media with purely imaginary roots. Mechanics of advanced materials and structures 16(1): 33-45.

[21] Guo L, Nie G and Chan C (2011) Elliptical inhomogeneity with polynomial eigenstrains embedded in orthotropic materials. Archive of Applied Mechanics 81(2): 157-170.

[22] Xu B-X and Wang M-Z (2007) The arithmetic mean property for rotational symmetrical inclusions with rotational symmetrical eigenstrains. ZAMM - Journal of Applied Mathematics and Mechanics/Zeitschrift für Angewandte Mathematik und Mechanik: Applied Mathematics and Mechanics 87(1): 59-69.

[23] Chen Y (2014) Solution for Eshelby's elliptic inclusion with polynomials distribution of the eigenstrains in plane elasticity. Applied Mathematical Modelling 38(19-20): 4872-4884.

[24] Zhou K and Hoh H J and Wang X, et al. (2013) A review of recent works on inclusions. Mechanics of Materials 60: 144-158.

[25] Cebeci H and de Villoria R G and Hart A J, et al. (2009) Multifunctional properties of high volume fraction aligned carbon nanotube polymer composites with controlled morphology. Composites Science and Technology 69(15-16): 2649-2656.

[26] Bradford P D and Wang X and Zhao H, et al. (2010) A novel approach to fabricate high volume fraction nanocomposites with long aligned carbon nanotubes. Composites Science and Technology 70(13): 1980-1985.

[27] Wardle B L and Saito D S and Garcia E J, et al. (2008) Fabrication and characterization of ultrahigh - volume - fraction aligned carbon nanotube - polymer composites. Advanced Materials 20(14): 2707-2714.

[28] Prabhu B and Suryanarayana C and An L, et al. (2006) Synthesis and characterization of high volume fraction $\mathrm{Al}-\mathrm{Al} 2 \mathrm{O} 3$ nanocomposite powders by high-energy milling. Materials Science and Engineering: A 425(1-2): 192-200.

[29] Li S, Sauer R and Wang G (2005) A circular inclusion in a finite domain I. The Dirichlet-Eshelby problem. Acta mechanica 179(1-2): 67-90.

[30] Wang G, Li S and Sauer R (2005) A circular inclusion in a finite domain II. The Neumann-Eshelby problem. Acta mechanica 179(1-2): 91-110.

[31] Li S, Sauer R A and Wang G (2007) The Eshelby tensors in a finite spherical domain - Part I: Theoretical formulations. Journal of applied mechanics 74(4): 770-783.

[32] Li S, Wang G and Sauer R A (2007) The Eshelby tensors in a finite spherical domain-Part II: applications to homogenization. Journal of Applied Mechanics 74(4): 784-797. 
[33] Gao X-L and Ma H (2010) Solution of Eshelby's inclusion problem with a bounded domain and Eshelby's tensor for a spherical inclusion in a finite spherical matrix based on a simplified strain gradient elasticity theory. Journal of the Mechanics and Physics of Solids 58(5): 779-797.

[34] Ma H and Gao X-L (2011) Strain gradient solution for a finite-domain Eshelby-type plane strain inclusion problem and Eshelby's tensor for a cylindrical inclusion in a finite elastic matrix. International Journal of Solids and Structures 48(1): 44-55.

[35] Mejak G (2011) Eshebly tensors for a finite spherical domain with an axisymmetric inclusion. European Journal of Mechanics-A/Solids 30(4): 477-490.

[36] Zou W-N, He Q-C and Zheng Q-S (2012) Inclusions in a finite elastic body. International Journal of Solids and Structures 49(13): 1627-1636.

[37] Zou W-N and He Q-C (2017) Eshelby's problem of a spherical inclusion eccentrically embedded in a finite spherical body. Proceedings of the Royal Society A: Mathematical, Physical and Engineering Sciences 473(2198): 20160808.

[38] Chen Y (2013) Solution for Eshelby's elastic inclusions in a finite plate using boundary integral equation method. Engineering Analysis with Boundary Elements 37(7-8): 1089-1094.

[39] Pan C and Yu Q (2014) Inclusion problem of a two-dimensional finite domain: The shape effect of matrix. Mechanics of Materials 77: 86-97.

[40] Pan C and Yu Q (2017) Investigation of an arbitrarily shaped inclusion embedded in a two-dimensional finite domain. International Journal of Mechanical Sciences 126: 142-150.

[41] Sharma P and Sharma R (2003) On the Eshelby's inclusion problem for ellipsoids with nonuniform dilatational Gaussian and exponential eigenstrains. Journal of applied mechanics 70(3): 418-425.

[42] Ma L and Korsunsky A M (2014) The principle of equivalent eigenstrain for inhomogeneous inclusion problems. International Journal of Solids and Structures 51(25-26): 4477-4484.

[43] Ma L, Wang B and Korsunsky A M (2015) Plane deformation of circular inhomogeneous inclusion problems with non-uniform symmetrical dilatational eigenstrain. Materials \& Design 86: 809-817.

[44] Mura T, 2013. Micromechanics of defects in solids Springer Science \& Business Media. 